\title{
Enfance
}

http://www.necplus.eu/ENF

Additional services for Enfance:

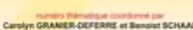

Email alerts: $\underline{\text { Click here }}$

Subscriptions: Click here

d'Anthony J. DeCasper

Commercial reprints: $\underline{\text { Click here }}$

Terms of use : $\underline{\text { Click here }}$

\section{Plasticité développementale dans le cortex auditif : La résultante de l'état de maturation cortical et des caractéristiques sonores de l'environnement}

Florian Occelli et Jean-Marc Edeline

Enfance / Volume 2017 / Issue 03 / September 2017, pp 329 - 348

DOI: 10.4074/S0013754517003044, Published online: 01 November 2017

Link to this article: http://www.necplus.eu/abstract_S0013754517003044

How to cite this article:

Florian Occelli et Jean-Marc Edeline (2017). Plasticité développementale dans le cortex auditif : La résultante de l'état de maturation cortical et des caractéristiques sonores de l'environnement. Enfance, 2017, pp 329-348 doi:10.4074/S0013754517003044

Request Permissions : $\underline{\text { Click here }}$ 


\title{
Plasticité développementale dans le cortex auditif : La résultante de l'état de maturation cortical et des caractéristiques sonores de l'environnement
}

\author{
Florian OCCELLI et Jean-Marc EDELINE
}

\begin{abstract}
RÉSUMÉ
Chez l'animal, la plasticité développementale du système auditif n'a été que sporadiquement étudiée jusqu'au début des années 2000. Depuis, plusieurs séries de travaux ont démontré l'existence d'une période critique néonatale pour le fonctionnement du cortex auditif et ont révélé les conséquences drastiques que peuvent avoir des environnements bruités au cours de cette période. Néanmoins, après la fin de cette période critique néonatale, la susceptibilité du système auditif aux environnements bruités reste encore élevée chez l'animal juvénile surtout si ces environnements présentent des caractéristiques acoustiques particulières qui ne sont pas présentes dans les milieux naturels.
\end{abstract}

MOTS-CLEFS : PlasticITÉ CORTICALE, PÉRIODE CRITIQUE, ÉLECTROPHYSIOLOGIE, CORTEX AUDITIF, ENVIRONNEMENT BRUITÉ, CARTE TONOTOPIQUE CORTICALE.

NeuroPSI, UMR CNRS 9197, Université Paris-Sud, Bat 446, 91405 Orsay. Emails : florian.occelli@u-psud.fr, jean-marc.edeline@u-psud.fr 


\begin{abstract}
ABSTRAC'T
In the auditory system, the developmental plasticity has only been studied sporadically until 2000. Since then, several lines of research have provided compelling evidence for the existence of a postnatal critical period in the auditory cortex, and have revealed the drastic consequences triggered by exposures to noisy environments. However, after this postnatal critical period, the auditory cortex of juveniles still remains sensitive to long-lasting exposures to noisy environments, especially when their acoustic characteristics fundamentally differ from those present in natural environments.

KEY-WORDS: CORTICAL PLASTICITY, CRITICAL PERIOD, ELECTROPHYSIOLOGY, AUDITORY CORTEX, NOISE EXPOSURE, TONOTOPIC CORTICAL MAP.
\end{abstract}




\section{CADRE GÉNÉRAL}

Les travaux d'Hubel et Wiesel au début des années 1960 ont révélé l'extraordinaire plasticité du système visuel lorsque des manipulations de l'environnement surviennent lors d'un stade particulier du développement appelé " période critique ». Initialement, ces travaux affirmaient que, passée cette étroite fenêtre temporelle, la plasticité des systèmes sensoriels étaient très limitée voire inexistante (voir pour revue, Wiesel, 1982). Curieusement, dans le système auditif, la plasticité corticale a d'abord été étudiée chez l'adulte et ce n'est que relativement récemment (depuis une quinzaine d'années) que des travaux ont fait le lien entre plasticité développementale et plasticité adulte. Cet article tentera de déterminer si ces phénomènes de plasticité se situent dans un continuum ou bien s'il y a lieu de les considérer comme deux domaines distincts.

\section{INTRODUCTION}

La plasticité des systèmes sensoriels est un vaste domaine qui regroupe des situations expérimentales extrêmement diverses allant de privations de tout ou partie des stimuli de l'environnement jusqu'à des tâches d'apprentissage perceptif qui requièrent parfois plusieurs mois d'entraînement (revue dans Weinberger, 1995). Dans le système visuel, les travaux d'Hubel et Wiesel au début des années 1960 ont fermement établi les bases de la plasticité développementale des systèmes sensoriels. Ces auteurs, puis leurs successeurs, ont démontré que des privations monoculaires, ou binoculaires, affectaient drastiquement la sélectivité des neurones pour la dominance oculaire ou l'orientation (revue dans Wiesel, 1982 ; Rauscheker 1991). Depuis, les mécanismes présidant à cette plasticité développementale ont été l'objet de myriades d'études qui ont successivement disséqué le rôle des systèmes neuromodulateurs (Bear \& Singer, 1986), des règles de plasticité Hebbiennes ${ }^{1}$ (Frégnac \& Shulz, 1999 ; Frégnac et al., 2010), des inhibitions GABAergiques ${ }^{2}$ (revue dans Hensch, 2005) et des différents types d'interneurones inhibiteurs corticaux (Fu et al., 2015 ; Larimer et al., 2016) dans la mise en place de cette plasticité développementale.

Au contraire, dans la modalité auditive, de nombreux auteurs ont initialement décrit des phénomènes de plasticité chez l'animal adulte dans des situations d'apprentissage comportemental (de type conditionnement classique), c'est-à-dire lorsque des stimuli acoustiques sont temporellement associés avec des renforcements appétitifs ou aversifs (voir pour revue, Edeline, 1999). À l'origine, ce type de travaux a décrit des augmentations de potentiels évoqués,

1 Ces règles, initialement proposées par Donald Hebb en 1949, décrivent comment la coïncidence entre l'activité pré et post-synaptique contribue à augmenter, ou au contraire à diminuer, l'efficacité d'une synapse (ou d'un groupe de synapses) entre 2 neurones (ou 2 groupes de neurones).

2 Le GABA est un neurotransmetteur qui inhibe les neurones quand il est libéré par une terminaison présynaptique. Les différents types d'interneurones corticaux (14 dans la classification de Ascoli et al., 2008) libèrent tous du GABA, mais certains libèrent également des peptides comme la somatostatine ou le VIP. 
de réponses multi-unitaires (d'un petit groupe de neurones), ou de réponses unitaires (d'un seul neurone) lorsqu'un son d'une fréquence donnée (5 kHz par exemple) était utilisé comme stimulus conditionnel systématiquement associé à l'occurrence d'un stimulus inconditionnel (un léger choc sur la patte de l'animal ou bien une boulette de nourriture). Les recherches ont pris une autre dimension à la fin des années 1980, lorsque plusieurs laboratoires ont montré, d'une part, que ces augmentations de réponses évoquées correspondaient à des changements sélectifs des champs récepteurs des neurones corticaux (par exemple, Bakin \& Weinberger, 1990 ; Edeline \& Weinberger, 1993) et, d'autre part, que ces effets étaient les prémices aux réorganisations de cartes corticales qui favorisent la représentation corticale des stimuli signifiants (par exemple, Recanzone et al, 1993). Depuis ces travaux initiaux, une vaste littérature a décrit les différentes situations comportementales (conditionnement classique ou instrumental, appétitif ou aversif) conduisant à des modifications du traitement des stimuli acoustiques au sein du système auditif adulte (voir pour revue Edeline, 2003, Weinberger 2004, Fritz et al., 2005). Il est important de préciser que des changements sélectifs des champs récepteurs corticaux en faveur d'un stimulus signifiant peuvent s'observer très rapidement, en quelques minutes et une dizaine d'essais d'entraînement comportemental (Edeline et al., 1993 ; Fritz et al., 2003), suggérant un rôle potentiel de l'attention dans les prémices à la plasticité des champs récepteurs corticaux. D’autres dimensions que la fréquence sonore peuvent être affectées par les effets d'un entraînement comportemental. Par exemple, lorsqu'un niveau particulier d'intensité sonore devient prédictif de l'arrivée d'un renforcement alimentaire, les neurones deviennent plus sensibles à cette intensité sonore indépendamment de la fréquence des sons utilisés (entre 2 et $28 \mathrm{kHz}$ dans l'expérience de Polley et al., 2004). Comme nous allons le voir, les travaux effectués dans le cadre de la plasticité développementale ont démarré relativement récemment précisant de mieux en mieux, au cours du développement du système auditif, la période critique et les conditions qui déclenchent des phénomènes de plasticité.

\section{Privations Auditives Au COURS DU DÉVElopPEMENT : VERS LA DÉFINITION DE LA PÉRIODE CRITIQUE?}

Initialement, au milieu des années 1980, des travaux avaient déjà suggéré que l'exposition de très jeunes animaux à des milieux acoustiquement appauvris ou enrichis avait un impact sur le développement de la fonction auditive. Par exemple, Sanes et Constantine Paton (1983) ont exposé de jeunes souris à un train de clics présenté à $20 \mathrm{~Hz}$ et $88 \mathrm{~dB}$ depuis l'âge de 8 jours jusqu'à l'âge de 20 jours après la naissance. Alors que les seuils du nerf auditif ne semblaient pas affectés par cette exposition, les auteurs ont rapporté un élargissement des champs récepteurs auditifs dans le colliculus inférieurs, suggérant que cette manipulation atténuait la sélectivité fréquentielle dès le tronc cérébral auditif. 
Curieusement, ce type d'étude n'a pas été poursuivi immédiatement et ce n'est qu'au milieu des années 2000, qu'une série de travaux du laboratoire de D. H. Sanes a testé les conséquences de privations auditives au cours du développement. Initialement, celles-ci ont été réalisées par des ablations totales des deux cochlées juste avant le début de l'audition (10 jours après la naissance chez la gerbille). Une à deux semaines plus tard, les auteurs ont échantillonné les neurones corticaux dans des conditions in vitro. En comparant les réponses obtenues chez des animaux exposés avec celles obtenues chez les animaux contrôles, une augmentation générale d'excitabilité des neurones corticaux a été mesurée chez les animaux exposés, ce qui implique à la fois des changements de la transmission glutamaergique (courants excitateurs plus larges et plus longs que chez les animaux contrôles) et des changements de la transmission GABAergique (courants inhibiteurs moins amples que chez les animaux contrôles ; voir par exemple, Kotak et al., 2005, 2008).

Pour se rapprocher de situations de surdité partielle pouvant survenir dans l'enfance ou l'adolescence humaine (lors d'otites sévères ou de trauma sonores) certaines expériences chez la gerbille adolescente ont consisté à réaliser une perte auditive partielle par désinsertion des muscles des deux oreilles moyennes provoquant une perte auditive de l'ordre de 40-50 dB pour toutes les fréquences sonores. Alors que les animaux présentaient une augmentation de leur seuil auditif de $40 \mathrm{~dB}$ et que leur aptitude à détecter des sons modulés en fréquence $(\mathrm{SAM})$ à basses fréquences $(5 \mathrm{~Hz})$ était moins bonne, la détection de SAM à fréquences plus élevées $(100 \mathrm{~Hz})$ n'était pas affectée dans un protocole d'évitement actif. Ainsi, si certaines propriétés des neurones corticaux sont affectées (seuils plus élevés, taux de décharge plus faibles), d'autres, comme la synchronisation temporelle avec la modulation d'amplitude, semblent peu ou non altérées (Rosen et al., 2012). Néanmoins, la moyenne des courbes neurométriques des neurones corticaux corrèle significativement avec les courbes audiométriques des animaux suggérant un lien étroit entre l'activité du cortex auditif et la tâche comportementale de détection des SAM. Cela indique aussi que l'impact de la privation acoustique sur l'activité du cortex auditif pourrait expliquer, au moins en partie, les difficultés d'animaux partiellement sourds.

Bien que très intéressantes, ces données ne constituent pas le pendant des travaux de Hubel et Wiesel dans le système visuel puisque des manipulations irréversibles des entrées auditives (ablations cochléaires ou privations auditives partielles) ont été utilisées. Les études plus récentes du laboratoire de D. H. Sanes se sont clairement rapprochées du paradigme original utilisé dans le système visuel. En privant temporairement et partiellement (par des bouchons d'oreilles qui atténuent les sons d'origine aérienne de $40 \mathrm{~dB}$ SPL, donc sans supprimer la conduction osseuse et la possibilité pour l'animal d'entendre ses propres bruits biologiques) des gerbilles d'entrées auditives pendant une brève période, de l'entrée en fonction de la cochlée jusqu'à la fin de la maturation cochléaire (12 jours à compter de 11 jours après la naissance), les auteurs ont pu tester dans quelle mesure une perturbation survenant au tout début de la fonction 
auditive modifie ultérieurement les performances comportementales des sujets. Des animaux testés 1 à 4 semaines après la fin de la privation auditive partielle ont montré des altérations des seuils de discrimination de la profondeur de modulation d'amplitude (Caras \& Sanes, 2015), ainsi que dans la perception de stimuli modulés en amplitude dans des milieux bruités (Ihlefeld et al., 2016). Le fait que ces altérations ne sont pas réversibles lorsque l'animal est remis dans des conditions sonores normales, et que, de plus, ces altérations ne surviennent pas si la privation se fait entre 23 et 35 jours postnatals, suggèrent clairement l'existence d'une période critique dans le développement du système auditif.

De tels résultats montrent donc que les entrées sensorielles ont un rôle fondamental dans le développement des capacités de résolution temporelle du système auditif à la base des perceptions de séquences sonores complexes.

De façon plus surprenante, et probablement pertinente par rapport aux problèmes développementaux présentés par l'enfant prématuré, ce laboratoire a pu montrer les interactions entre la période critique du développement du système visuel et celle du système auditif. Sachant que la maturation structurale et fonctionnelle du système auditif commence avant celle du système visuel, est-il possible que la maturation d'un de ces deux systèmes influence la maturation de l'autre? En avançant ou en retardant l'expérience visuelle, Mowery et al. (2016) ont étudié les conséquences de l'activation de l'entrée visuelle au cours de la période critique de développement du système auditif. Avancer le fonctionnement de la vision, c'est-à-dire induire le système visuel à fonctionner prématurément, conduit à fermer la période critique de développement du système auditif. De ce fait, une perte auditive temporaire, qui normalement devrait avoir des conséquences sur les propriétés des neurones du cortex auditif, reste sans conséquence. Inversement, le fait de retarder l'entrée en fonction du système visuel peut étendre la période critique du système auditif de plusieurs jours. Le résultat le plus impressionnant de ce travail est que, lorsqu'une expérience visuelle très précoce est couplée à un déficit auditif temporaire, dont normalement les animaux récupèrent, des effets sur la circuiterie inhibitrice $\mathrm{du}$ cortex auditif peuvent persister chez l'adulte (Mowery et al., 2016). Ces données suggèrent donc que si la séquence naturelle de mise en place des fonctions sensorielles est perturbée, comme c'est très probablement le cas chez les enfants grands prématurés, la période critique de plasticité développementale de l'audition pourrait être modifiée et peut conduire à des altérations stables. Il est bien sûr difficile d'extrapoler les données obtenues sur la période critique de rongeurs au développement des systèmes sensoriels de l'enfant prématurés, qui sont en général connu pour présenter des profils sensoriels atypiques tant du point de vue comportemental (Wickremasinghe et al., 2013) que des rythmes électroencéphalographiques déclenchés par les stimuli sensoriels (André et al., 2010 ; Vanhatalo et al., 2005). En fait certaines unités de soins intensifs pour nouveau-nés semblent déjà avoir pris en compte la fragilité potentielle du système auditif des prématurés (voir pour revue McMahon et al., 2012). 


\section{EXPOSITIONS À DES ENVIRONNEMENTS BRUITÉS : UN CONTINUUM DE PLASTICITÉ DE L'ANIMAL EN DÉVELOPPEMENT À L'ADULTE?}

Chez l'homme, comme chez l'animal, la limite entre une exposition sensorielle sans danger et une exposition à risque est relativement difficile à déterminer. Il est traditionnellement admis que des pressions sonores de $85 \mathrm{~dB}$ ne provoquent ni perte auditive permanente ni perte auditive temporaire ; ce qui justifie le seuil de $85 \mathrm{~dB}$ maximum d'exposition admis en milieu professionnel. Des pressions sonores supérieures à $110 \mathrm{~dB}$ sont connues pour provoquer des dommages irréversibles au niveau de la cochlée, à la fois au niveau des cils des cellules ciliées et au niveau des synapses entre cellules ciliées et fibres du nerf auditif. Des pressions sonores entre 85 et $110 \mathrm{~dB}$ sont quant à elles supposées provoquer des pertes auditives temporaires qui peuvent cependant avoir des conséquences à très long terme, par exemple en provoquant un vieillissement accéléré des circuits auditifs centraux (pour revue, voir Gourévitch et al., 2014). Notons qu'un second paramètre est aussi nécessaire à prendre en compte : la durée de l'exposition. Pour simplifier, si une intensité d'environ $90 \mathrm{~dB}$ est sans danger sur une période de quelques secondes, elle peut s'avérer lésante sur une période de quelques heures.

Pour ce qui est des effets des environnements bruyants ne provoquant ni perte auditive permanente, ni perte temporaire, la question est tout aussi complexe. En effet, il faut considérer que les expositions à des intensités sonores non-traumatisantes ont été faites sur des animaux à des stades de maturation très variés, allant des stades très précoces du développement postnatal à des animaux adolescents, et à des animaux adultes. Il est nécessaire de garder à l'esprit ces différents stades développementaux lorsque nous examinons les différents résultats de la littérature.

Certaines espèces de mammifères naissent avec des systèmes sensoriels prêts à fonctionner de façon adaptée. C'est le cas du cobaye et des primates, dont l'Homme. D'autres espèces, comme le rat ou la souris, naissent avec plusieurs systèmes sensoriels encore non-fonctionnels, en particulier l'audition. Cette particularité est une chance extraordinaire d'étudier la mise en place de l'audition dans des conditions privilégiées d'accès à l'expérimentation hors de l'utérus maternel. Mais, au-delà de la mise en place d'un système sensoriel, il est possible ainsi d'étudier finement l'influence des environnements acoustiques sur cette mise en place. Les premières réponses auditives sont enregistrables chez le rat au jour 11 postnatal (P11) (Cole \& Robertson, 1992 ; Zhang et al., 2001). Au début des années 2000, on a mis en évidence une perturbation majeure survenant dans le système auditif immature s'il est exposé à du bruit durant sa période critique (Zhang et al., 2001). En exposant des ratons à P9 (9 jours après la naissance), soit 2 jours avant l'entrée en fonction de la cochlée, lorsque le système auditif n'est pas opérationnel à des sons purs (4 ou $19 \mathrm{kHz}$ ) à $70 \mathrm{~dB}$, ces fréquences 
sont surreprésentées dans la carte tonotopique corticale ${ }^{3}$, effet qui se maintient jusqu'à 80 jours postnatals. En outre, les champs récepteurs corticaux pour ces fréquences sont plus larges que la normale, laissant supposer que la sélectivité fréquentielle des neurones est amoindrie. Afin de valider l'hypothèse selon laquelle l'environnement acoustique joue un rôle majeur dans la mise en place de la carte tonotopique corticale, des ratons au stade P7 ont été exposés à du bruit blanc $^{4}$ à $70 \mathrm{~dB}$ pendant 24 heures sur 24 (Chang \& Merzenich, 2003). L'impact sur le système auditif a été évalué en suivant l'évolution de la carte tonotopique corticale à P16, P26 et P50. Chez les animaux élevés de façon standard le gradient tonotopique s'établit progressivement pour aboutir à P50 à une organisation tonotopique proche de celle de l'adulte. Chez les animaux exposés, aucun gradient tonotopique n'apparaît à P50. Au contraire, une surreprésentation des hautes fréquences et une sous-représentation des basses fréquences s'établissent dans un ordre topographique qui est indépendant de la position anatomique habituelle au sein de l'aire correspondant au cortex auditif primaire (Figure 1). Notons aussi que les champs récepteurs des animaux exposés sont plus larges à P50 que ceux des animaux non-exposés de même âge.

Les auteurs ont fait l'hypothèse que, chez les animaux exposés durant la période critique, il existe une période étendue de plasticité du cortex auditif. Pour tester cette hypothèse, de nouveaux animaux sont exposés à des bruits blancs comme précédemment jusqu'à P50, puis à des sons de $7 \mathrm{kHz}$ jusqu'à P74. Ces animaux doublement exposés (à des bruits blancs puis à des pulses) sont comparés à des animaux simplement exposés à des pulses entre P50 et P74. Aucun effet de l'exposition à des pulses de $7 \mathrm{kHz}$ entre $\mathrm{P} 50$ et P74 n'est observé. En revanche chez des animaux exposés au bruit blanc durant leur période critique, on voit apparaître dans la carte corticale une plus grande représentation des fréquences autour de $7 \mathrm{kHz}$, qui, de plus, semble présenter un léger gradient tonotopique. Notons que l'on retrouve cette surreprésentation des fréquences moyennes jusqu'au stade adulte. Cela est d'autant plus intéressant que le gradient tonotopique semble se mettre en place avec des proportions normales entre les basses, moyennes et hautes fréquences à P120 si l'on ne réalise pas cette exposition supplémentaire.

Le décours temporel de cette période critique va être précisé par des études ultérieures de Villers-Sidani et al. (2007, 2008). De l'ensemble de ces études, on peut tirer deux principales conclusions. Premièrement, chez le rat, la période critique stricto sensu s'étend de P11 à P13 et on peut la définir comme la période au cours de laquelle une surexposition à des stimuli acoustiques peut entraîner une perturbation majeure de la maturation du réseau cortical. Deuxièmement,

3 Chez toutes les espèces l'organisation présente dans la cochlée se retrouve au niveau du cortex auditif : on trouve toujours un gradient des fréquences préférées des neurones des hautes vers les basses fréquences au sein de l'aire auditive primaire.

4 Le bruit blanc est un bruit de large spectre qui couvre toutes les fréquences audibles à une même pression sonore. 

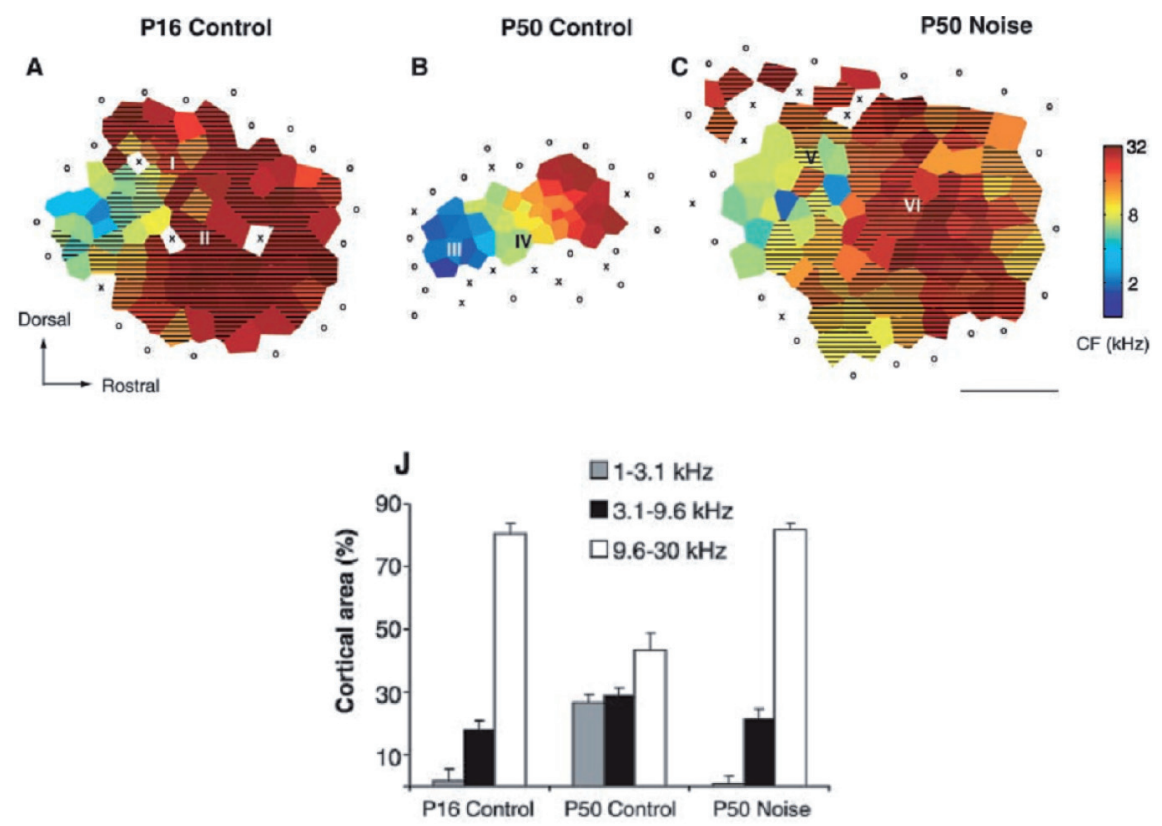

\section{Figure 1.}

Exemples de cartes corticales individuelles chez un rat contrôle à P16 (A), un rat contrôle à P50 (B) et chez un rat exposé à P50 (C). Pourcentage du cortex auditif primaire couvert par les différentes bandes de fréquences à P16 et P50 chez les rats non exposés (contrôles) et à $\mathrm{P} 50$ chez les rats exposés (J). Extrait de la thèse de F. Occelli (2015), modifié d’après Chang et Merzenich, 2003.

cette période, qui par définition est sensible à la composition de l'environnement sonore, peut ne pas être homogène le long de la carte corticale. C'est-à-dire qu'il est possible, en fonction de l'environnement acoustique de voir des zones correspondant à certaines plages de fréquences atteindre leur maturité à des vitesses différentes. Ainsi, en correspondance avec l'environnement acoustique auquel les animaux étaient exposés, certains territoires corticaux présentaient de forts signes d'immaturité (moins de cellules inhibitrices, moindre capacité de suivi d'enveloppes temporelles, et plus grande largeur de bande des champs récepteurs), alors même que d'autres territoires, au sein des mêmes aires corticales et sur les mêmes animaux, présentaient des réponses évoquées matures. De telles données sont importantes lorsque l'on considère que les enfants grands prématurés dans leur incubateur sont essentiellement stimulés par des bruits de machine dont la composition peut s'apparenter à du bruit blanc, puisque ce sont des bruits continus à très large bande. Des protocoles de stimulation naturels (bruits maternels comme la voix ou des battements cardiaques) ont été tentés pour mimer un environnement plus compatible avec le développement normal des systèmes (voir par exemple Webb et al., 2015). 
Les travaux montrent également que cette forte sensibilité du système auditif à l'environnement sonore persiste bien au-delà de cette période critique. Cela a été bien décrit dans des expériences effectuées chez de jeunes chats dont les réponses corticales semblent pourtant comparables à celles observées chez des chats adultes. Des chatons juvéniles de 2 à 7 mois (avant la maturité sexuelle) ont été exposés à un ensemble de sons purs de $50 \mathrm{~ms}$ entre 5 et $20 \mathrm{kHz}$ présentés à $80 \mathrm{~dB}$ SPL en continu pendant 3-4 mois (Norena et al., 2006). En comparant les réponses corticales des animaux exposés à celles obtenues chez des animaux d'âge équivalent élevés dans une animalerie standard, une chute drastique du taux de décharge évoqué a été notée en réponse à des fréquences de sons purs correspondant à la plage de fréquences de l'exposition acoustique (figure 2). Cette plasticité s'observe bien qu'aucune modification des audiogrammes ne soit détectable (à partir des réponses évoquées du tronc cérébral), suggérant une absence de perte auditive périphérique. L'ensemble aboutit donc à une
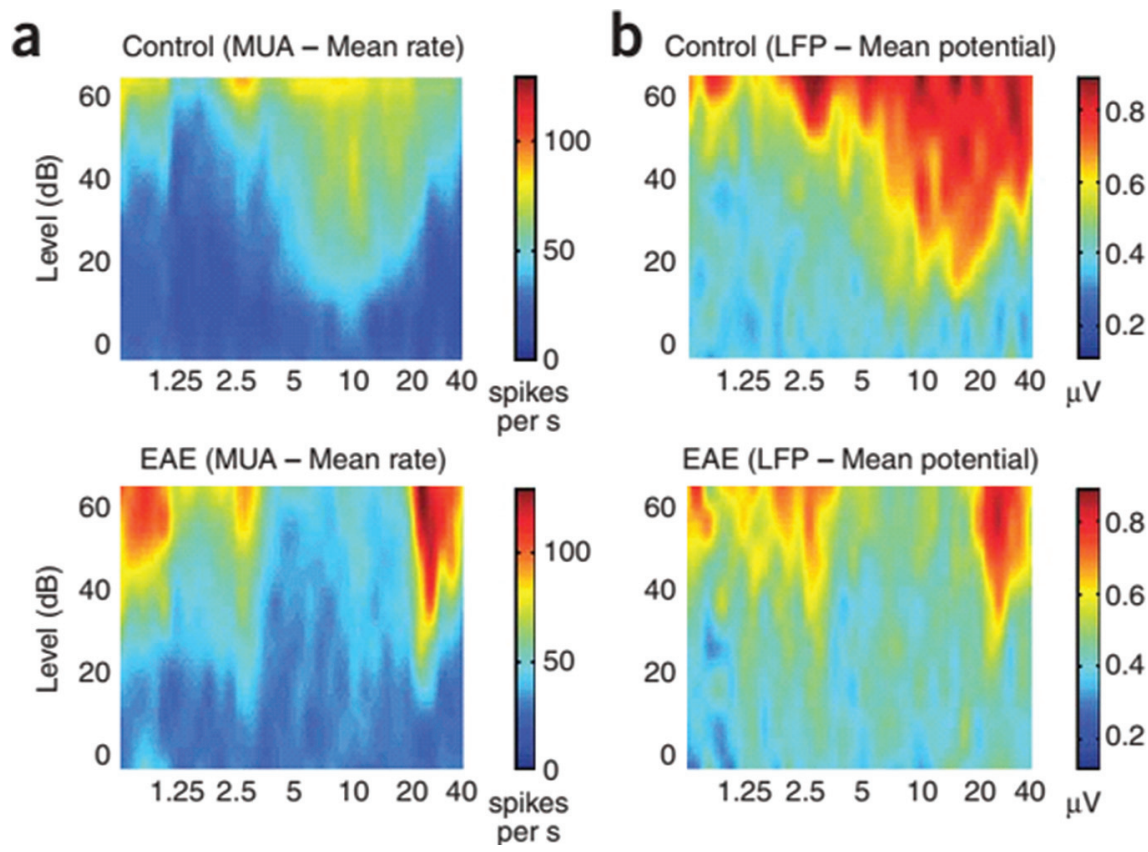

Figure 2.

Réponses évoquées moyennes du cortex auditif en fonction de la pression sonore et de la fréquence du stimulus auditif, obtenues à partir des décharges neuronales (MUA, à gauche) et du potentiel de champs local (LFP, à droite) chez des animaux contrôles (notés « Control » en haut) ou exposés à un milieu bruité (notés EAE, en bas). Notez l'absence totale de réponses à la fois sur les décharges des neurones (MUA) et du potentiel de champs local (LFP) chez les animaux exposés (en bas) par rapport aux animaux contrôles (en haut). Extrait de la thèse de F. Occelli (2015, modifié d'après Noreña et al., 2006). 
perturbation claire de la carte tonotopique corticale en l'absence de modification périphérique (au niveau de la cochlée).

Un effet moindre, mais comparable, a été décrit avec des expositions de seulement $68 \mathrm{~dB}$ SPL durant 6 semaines au lieu de $80 \mathrm{~dB}$ SPL durant 4 mois (Pienkowski \& Eggermont, 2009). Cet effet sur le taux de décharge évoqué, observé sur des animaux juvéniles, est réversible en 12 semaines après l'arrêt de l'exposition alors que carte la tonotopique corticale reste, quant à elle, altérée. Cet effet se retrouve même en diminuant l'exposition à 12 heures par jour durant 6 semaines (Pienkowski \& Eggermont, 2010a). En exposant pendant 10 semaines des chatons d'âges équivalents (2 à 7 mois) 24 heures par jour à deux bandes de bruit d'un tiers d'octave autour de 4 et de $16 \mathrm{kHz}$ à $72 \mathrm{~dB}$ SPL, on observe une réduction du taux de décharge dans les bandes de fréquences d'exposition comparable à celle des expériences précédentes, ainsi qu'une perturbation forte de la carte tonotopique corticale (Pienkowski \& Eggermont, 2010). Cette réduction de la réponse évoquée débute dès la première semaine d'exposition et s'étend progressivement à l'ensemble des territoires concernés en 4 semaines (Pienkowski et al., 2011). Suite à ces expositions à des environnements bruités, des effets ont également été détectés dans le cortex auditif secondaire.

Pourtant, une dernière étude sur la période de développement faisant suite à la période critique au cours de la maturation cochléaire vient nuancer les effets décrits ci-dessus. Dans cette étude, plutôt que d'utiliser des sons purs ou des bandes de bruits, des chatons d'environ 3 mois ont été exposés à des enregistrements de sons complexes provenant d'une usine et d'un chantier de construction (Pienkowski et al., 2013). Cet environnement sonore s'étend à l'ensemble des fréquences du spectre auditif du chat en deçà de $16 \mathrm{kHz}$. L'exposition a été continue pendant une période située entre 8 et 15 semaines. En comparaison des effets massifs observés avec des sons purs, ces sons complexes n'ont produit aucun effet significatif sur le taux de décharge évoqué par rapport aux chats contrôles (Figure 4).

\section{ENVIRONNEMENT ENRICHI VERSUS ENVIRONNEMENT BRUITÉ ?}

D’une façon surprenante, la distinction entre « environnement enrichi » et « environnement bruité » n’a pas toujours été faite dans la littérature électrophysiologique décrivant les effets d'exposition d'animaux matures ou immatures sur l'organisation fonctionnelle et les propriétés des neurones du cortex auditif. Il est d'ailleurs amusant de constater que la composition spectrale de séquences extraites de symphonies classiques n'est pas très différente de celles de bruits industriels.

À la croisée de ces deux types d'environnements, une étude particulièrement informative a utilisé un environnement enrichi unique en son genre (Engineer et al., 2004). La particularité de ce dispositif est qu'en se déplaçant dans la cage un animal déclenchait par l'intermédiaire d'un détecteur de mouvements, un son différent à chaque emplacement. Les animaux, dits " exposés », ont été placés, 


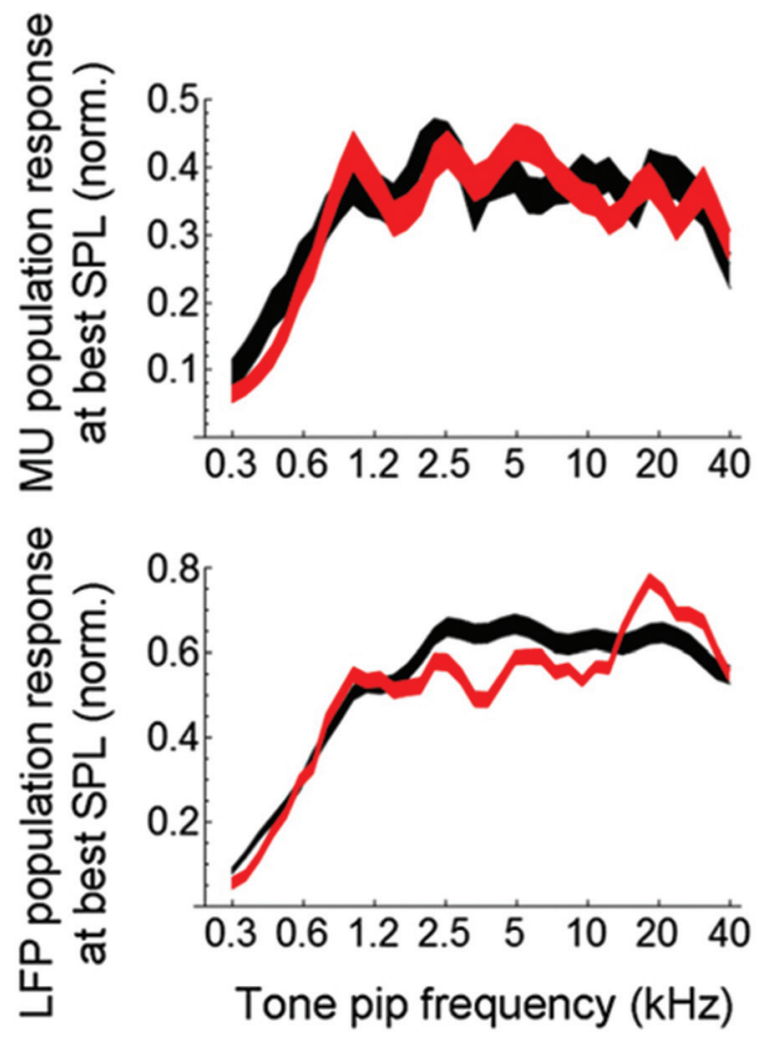

- Control (nMU = 961; nLFP = 1458)

Factory noise EAE (nMU = 693; nLFP = 1012)

\section{Figure 3.}

Réponses évoquées normalisées et moyennées obtenues à partir des décharges neuronales (MU en haut) pour les chats non-exposés (en noir) ou exposés à des enregistrements d'environnements acoustiques industriels (en rouge) ou à partir du potentiel de champs local (LFP en bas). Que ce soit sur la base des décharges neuronales (MU) ou du potentiel de champs local (LFP), aucune différence significative n'a été rapportée entre animaux contrôles et exposés. Extrait de la thèse de F. Occelli (2015, modifié d'après Pienkowski et al., 2013).

par groupe de 4 à 8 , dans cet environnement durant 8 semaines, alors que les animaux non-exposés (contrôles) sont hébergés par deux dans de petites cages conventionnelles.

La période d'exposition va de la $4^{\mathrm{e}}$ à la $12^{\mathrm{e}}$ semaine postnatale, soit également au-delà de la période critique précédemment définie. À la $12^{\mathrm{e}}$ semaine, au sortir de l'animalerie enrichie, des enregistrements électrophysiologiques ont été réalisés dans le cortex auditif. Les réponses évoquées sont enregistrées à la présentation de sons purs de différentes fréquences et intensités. Il en ressort que l'exposition 


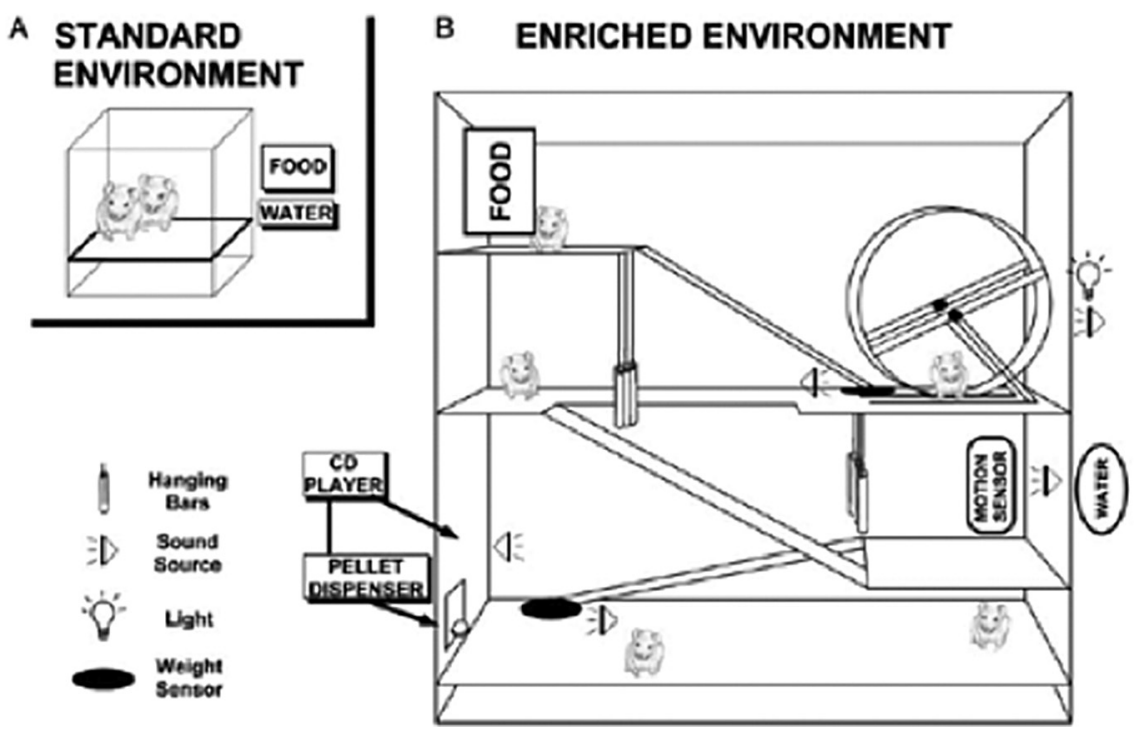

Figure 4.

Dispositif expérimental d'enrichissement auditif (B) par comparaison avec la cage d'élevage standard (A). Extrait de la thèse de F. Occelli (2015), modifié d'après Engineer et al. 2004.

à cet environnement n'affecte pas la carte tonotopique corticale. En revanche, les neurones corticaux des rats exposés sont plus sensibles aux stimuli acoustiques (quelle que soit leur fréquence) que ceux des animaux non-exposés ; en effet, le taux de décharge évoqué est plus important (de 20 à 30 \%), la largeur des champs récepteurs est augmentée et la durée des réponses est plus longue chez les rats exposés.

Ultérieurement, on a pu montrer que ce milieu enrichi altérait aussi la dynamique temporelle des réponses dans le cortex auditif (Percaccio et al., 2005). En présentant des sons purs ou des bruits blancs à différentes cadences, les neurones enregistrés chez les animaux exposés au milieu enrichi répondaient mieux aux cadences lentes $(<10 \mathrm{~Hz}$ ) et moins bien aux cadences rapides $(>10 \mathrm{~Hz})$. En termes de mécanismes, ce milieu enrichi influe sur les courants potentiels post-synaptiques excitateurs (tant sur l'amplitude que sur le décours temporel) mais pas sur les courants potentiels post-synaptiques inhibiteurs des neurones des couches 2 et 3 du cortex auditif, couches qui sont riches en interneurones inhibiteurs GABAergiques (Nichols et al., 2007).

Notons cependant qu'en dehors de la période critique néonatale classique, une sensibilité du système auditif existe aussi chez l'animal qui n'a pas encore atteint le stade adulte. Récemment, deux expériences effectuées chez de jeunes rongeurs (rats âgés de 3 mois ou moins) sont venues corroborer l'hypothèse selon laquelle l'exposition à des milieux « bruités » à l'adolescence pouvait perturber l'organisation du cortex auditif. 


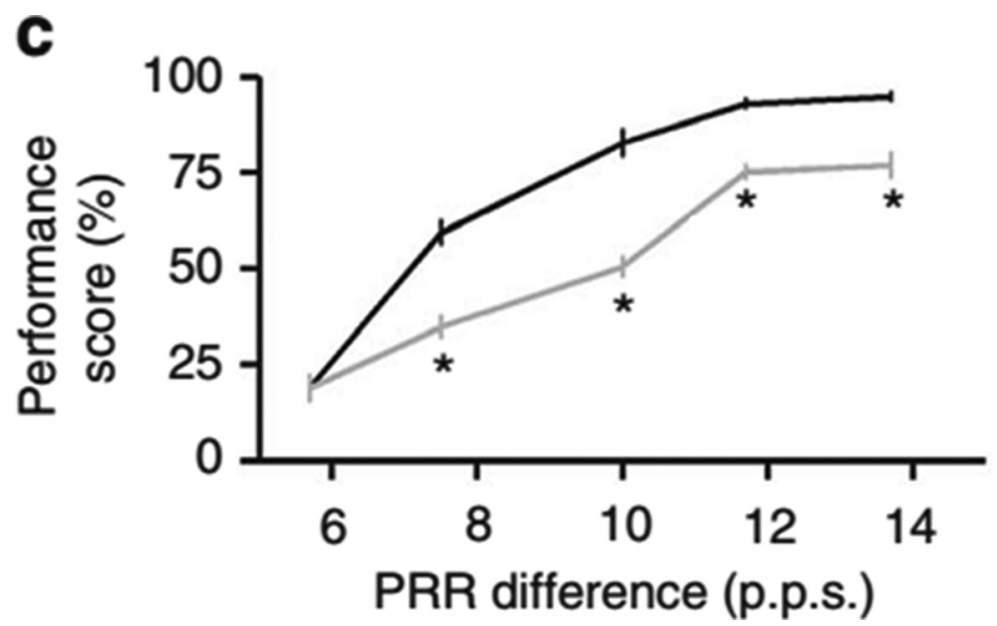

Figure 5.

Pourcentage moyen de bonnes réponses lors de la tâche comportementale en fonction de la différence en $\mathrm{Hz}$ (noté pps ici) entre les cadences à discriminer pour les animaux non-exposés (en noir) et exposés (en gris). Extrait de la thèse de F. Occelli (2015), modifié d'après Zhou et Merzenich., 2012.

Une première expérience a consisté à exposer, pendant 2 mois, des rats âgés de 3 mois à un bruit $(65 \mathrm{~dB})$ à large bande $(0,8$ à $30 \mathrm{kHz})$ constitué de pulses de $50 \mathrm{~ms}$ présentés en continu $(24$ heures $/ 24)$ à des cadences à la fois lentes et rapides allant de 3 à $18 \mathrm{~Hz}$ (Zhou \& Merzenich 2012). Suite à cette exposition, les animaux ne semblent pas avoir de perte auditive (d'après les potentiels évoqués du tronc cérébral). Pourtant au niveau cortical, les champs récepteurs sont plus larges pour toutes les fréquences sonores chez les animaux exposés que chez les non exposés, et les neurones des animaux exposés suivent des cadences moins élevées que ceux des animaux non-exposés (dans toute la gamme de fréquences du bruit). Des effets comparables ont été mis en évidence en exposant les animaux seulement 10 heures par jour, au lieu de 24 heures, que la cadence de présentation des stimuli ait été fixe (à 5 ou à $15 \mathrm{~Hz}$ ) ou variable. Chez les animaux exposés, une moindre proportion d'un type particulier d'interneurone inhibiteur cortical a été détectée. Ce type d'interneurone inhibiteur étant suspecté de jouer un rôle important dans la synchronisation des réponses avec les stimuli auditifs, ce résultat laisse entrevoir un mécanisme pour expliquer les effets sur le suivi des cadences dans le cortex auditif primaire.

Au cours de cette même étude, la capacité de discrimination entre différentes cadences a été testée comportementalement au cours d'une tâche de conditionnement opérant avec un renforcement appétitif. Dans ce protocole, les animaux non-exposés ont été capables de discriminer entre des cadences plus proches que les animaux exposés, indiquant un effet délétère de l'exposition auditive sur la capacité à discriminer des cadences. 
Dans une seconde expérience, des rats ont été exposés en continu à un bruit à large bande (4-45 kHz) à $70 \mathrm{~dB}$ pendant un mois (Zheng 2012). Ce qui correspond à un masquage de nombre de bruits présents dans l'environnement de l'animal. Le gradient tonotopique rostro-caudal allant des hautes aux basses fréquences qui s'observe chez les animaux contrôles n'est plus présent chez les animaux exposés. De plus, une remontée des seuils des potentiels évoqués corticaux d'environ $10 \mathrm{~dB}$ a été observée chez les rats exposés. Une partie des animaux a été testée dans une tâche comportementale de discrimination fréquentielle entre 2 sons purs de plus en plus proches. En absence de bruit, les animaux contrôles (non-exposés) ont été capables de discriminer des fréquences plus proches que les animaux exposés. En revanche, de façon surprenante, en présence de bruit, les animaux exposés montrent une capacité de discrimination plus fine que les animaux non exposés, suggérant ainsi que l'exposition prolongée au milieu bruité permet ultérieurement aux animaux d'être plus performants en présence de bruit, et ceci bien que leur cortex auditif présente une altération de la tonotopie. Cela suggère-t-il l'intervention de processus attentionnels, moins sollicités dans l'animalerie mais adaptés, voire nécessaires dans un environnement bruyant?

\section{QUE PEUT-ON CONCLURE DE L'ENSEMBLE DE CETTE LITTÉRATURE?}

La littérature électrophysiologique, obtenue chez le rongeur, décrivant les conséquences d'expositions au cours du développement à des environnements bruités (sans provoquer de perte auditive) apporte des informations cruciales. Elle confirme que la période critique est une période de vulnérabilité durant laquelle l'environnement peut avoir un effet néfaste important en un temps très limité. Quelques jours d'exposition à un environnement bruité continu suffisent pour perturber de façon prolongée la maturation de la carte auditive corticale. Cependant, au-delà de cette période critique, lorsque l'animal n'a pas encore atteint le stade de développement adulte, la susceptibilité du système auditif à l'environnement n'en est pas moins terminée. Par exemple, l'amplitude des réponses corticales peut être profondément réduite, de façon durable, suite à une exposition prolongée à un environnement bruité survenant lors de l'adolescence (au-delà de la période critique). Enfin, le contenu spectral et temporel de l'environnement acoustique semble avoir une importance non négligeable sur la nocivité des environnements bruités. Ainsi, alors que des sons purs délivrés à cadence rapide produisent un effet délétère sur les réponses évoquées dans le cortex auditif, des environnements acoustiques aux contenus plus riches et complexes n'ont pas, à ce jour, induit de tels effets.

Chez l'animal adolescent, il semble aussi que, même sans perte auditive temporaire, l'exposition de longue durée à un environnement sonore d'intensité modérée (70-85 dB SPL) soit délétère pour le système auditif. Néanmoins, rappelons que les expériences mettant en œuvre un environnement enrichi sur le plan acoustique, décrivent une facilitation des réponses corticales. 
En se basant sur des travaux récents traitant de la plasticité développementale du système visuel, des hypothèses peuvent être émises sur les mécanismes cellulaires sous-tendant ces phénomènes de plasticité, apparemment contradictoires, survenant à différents moments du développement. Par exemple, dans le système visuel, des privations monoculaires durant la période critique néonatale affectent particulièrement certains types d'interneurones inhibiteurs, les interneurones paravalbumines et somatostatine positifs (Kannan et al., 2016). Dans le cortex visuel, ces deux sous-populations sont sous le contrôle d'un troisième type d'interneurones appelé "interneurones VIP positifs » (Pi et al., 2013). Or, on a récemment montré que l'activation de cette dernière sous-population pouvait rouvrir une fenêtre de plasticité à l'âge adulte (Pi et al., 2013). Potentiellement, cela laisse entrevoir la possibilité que de futurs travaux puissent corriger des altérations du fonctionnement du système auditif survenues lors de la période critique néonatale, ou à l'adolescence, en réinstaurant une fenêtre de plasticité similaire à celle de la période critique néonatale, et ce grâce à une intervention ciblant sélectivement certaines populations d'interneurones corticaux.

De ce fait, on peut proposer que la plasticité induite dans le cortex auditif par des environnements bruités pourrait être due à la combinaison d'au moins deux facteurs. Le premier, le plus intuitif, est l'état de maturité fonctionnelle du cortex auditif qui est évidemment dépendante de l'âge des sujets. Le second est constitué par les différents paramètres des stimuli auditifs présents dans le milieu bruité (par exemple, la largeur de bande de l'environnement acoustique). En fonction des paramètres acoustiques des stimuli sonores, les sous-populations d'interneurones GABAergiques seront plus ou moins activées et, selon l'âge auquel se fait cette activation, les effets seront plus ou moins importants. Si cette hypothèse bâtie sur la littérature de ces 15 dernières années se trouvait validée, nous ferions face à une véritable révolution. En effet, dans la littérature humaine et animale, jusqu'à présent le dogme a toujours été que le danger d'un environnement acoustique venait de la combinaison de l'intensité sonore et de la durée de l'exposition. C'est sur ce dogme qu'ont été établies les législations sur les expositions acoustiques dans la plupart des pays de l'OCDE.

Notons que les effets de l'exposition aux bruits générés à l'intérieur des incubateurs dans lesquels sont placés les enfants prématurés ont été très étudiés il y a plus de 40 ans. Des recherches chez de très jeunes animaux ont aidé à mieux comprendre les effets délétères des niveaux sonores des incubateurs utilisés à cette époque (voir par exemple Douek et al., 1976). En effet, l'enfant de grande et moyenne prématurité ne bénéficie pas de la protection assurée par le système efférent au niveau de la chaîne tympano-ossiculaire et l'activité évoquée par les stimulations sensorielles est assez atypique (Milh et al., 2007 ; Colonnese et al., 2010 ; Chipaux et al., 2013). De ce fait, les unités de soins intensifs pour prématurés contrôlent rigoureusement les niveaux sonores admissibles (inférieurs à $60 \mathrm{~dB}$ SPL, voir pour revue McMahon et al., 2012), ce bien qu'il ne faille non plus tomber dans des situations qui s'apparenteraient à de la « privation 
sensorielle » (voir Pineda et al., 2014). Au vu des résultats de la littérature présentée ici, il semble indispensable de poursuivre l'effort de recherche sur les interactions entre l'âge des sujets exposés et les caractéristiques acoustiques du milieu bruité (largeur spectrale du bruit et cadence temporelle par exemple) afin d'affiner les réglementations. Au cours de ces dernières années, l'augmentation spectaculaire des troubles de l'audition pour toutes les tranches d'âges, y compris les plus jeunes, alors même que la législation est de plus en plus contraignante, doit nous inciter à plus de prudence.

\section{REMERCIEMENTS}

Nous tenons à remercier Carolyn Granier-Deferre et Benoist Schaal pour leur relecture attentive et leurs commentaires très pertinents qui ont contribué à améliorer cet article. Des remerciements spéciaux à Jessica Dubois pour les informations précieuses sur le développement des systèmes sensoriels chez les prématurés.

\section{RÉFÉRENCES}

André, M., Lamblin, M. D., d'Allest, A. M., Curzi-Dascalova, L., MoussalliSalefranque, F. S., Nguyen The, T., Vecchierini-Blineau, M. F., Wallois, F., Walls-Esquivel, E., \& Plouin, P. (2010) Electroencephalography in premature and full-term infants. Developmental features and glossary. Neurophysiol Clin., 40(2):59-124.

Ascoli, G. A. et al. (2008) Petilla terminology: nomenclature of features of GABAergic interneurons of the cerebral cortex. Nat Rev Neurosci. 9(7):557-68.

Bakin, J. S., \& Weinberger, N. M. (1990) Classical conditioning induces CS-specific receptive field plasticity in the auditory cortex of the guinea pig. Brain Res., 536(12):271-86.

Bear, M. F., \& Singer, W. (1986) Modulation of visual cortical plasticity by acetylcholine and noradrenaline. Nature, 320(6058):172-6.

Caras, M. L., \& Sanes, D. H. (2015) Sustained Perceptual Deficits from Transient Sensory Deprivation. J Neurosci., 35(30):10831-42.

Chang, E. F., \& Merzenich, M. M. (2003). Environmental noise retards auditory cortical development. Science, 300, 498-502.

Chipaux, M., Colonnese, M. T., Mauguen, A., Fellous, L., Mokhtari, M., Lezcano, O., Milh, M., Dulac, O., Chiron, C., Khazipov, R., \& Kaminska, A. (2013) Auditory stimuli mimicking ambient sounds drive temporal "delta-brushes" in premature infants. PLoS One, 8(11):e79028.

Cole, K. S., \& Robertson, D. (1992). Early efferent innervation of the developing rat cochlea studied with a carbocyanine dye. Brain Res, 575, 223-230.

Colonnese, M. T., Kaminska, A., Minlebaev, M., Milh, M., Bloem, B., Lescure, S., Moriette, G., Chiron, C., Ben-Ari, Y., \& Khazipov, R. (2010) A conserved switch in sensory processing prepares developing neocortex for vision. Neuron, 67(3):480-98. 
de Villers-Sidani, E., Chang, E. F., Bao, S., \& Merzenich, M. M. (2007). Critical period window for spectral tuning defined in the primary auditory cortex (A1) in the rat. J Neurosci, 27, 180-189.

de Villers-Sidani, E., Simpson, K. L., Lu, Y.-F., Lin, R. C. S., \& Merzenich, M. M. (2008). Manipulating critical period closure across different sectors of the primary auditory cortex. Nat Neurosci, 11, 957-965.

Douek, E., Dodson, H. C., Bannister, L. H., Ashcroft, P., \& Humphries, K. N. (1976) Effects of incubator noise on the cochlea of the newborn. Lancet, 2(7995): 1110-3.

Edeline, J.-M. (2003). The thalamo-cortical auditory receptive fields: Regulation by the states of vigilance, learning and the neuromodulatory systems. Experimental Brain Research, 153, 554-572.

Edeline, J-M (1999). Learning-induced physiological plasticity in the thalamo-cortical sensory system: A critical evalutation of receptive field plasticity and maps changes and their potential mechanisms. Progress in Neurobiolology, 57, 165-224.

Edeline, J.-M. \& Weinberger, N. M. (1993). Receptive field plasticity in the auditory cortex during frequency discrimination training: selective retuning independent of task difficulty. Behavioral Neuroscience, 107, 82-103.

Edeline, J.-M., Pham, P. \& Weinberger, N. M. (1993). Rapid development of learning-induced receptive field plasticity in the auditory cortex. Behavioral Neuroscience, 107, 539-551.

Engineer, N. D., Percaccio, C. R., Pandya, P. K., Moucha, R., Rathbun, D. L., \& Kilgard, M. P. (2004). Environmental enrichment improves response strength, threshold, selectivity, and latency of auditory cortex neurons. I Neurophysiol, 92, 73-82.

Frégnac, Y., Pananceau, M., René, A., Huguet, N., Marre, O., Levy, M., \& Shulz, D. E. (2010) A Re-Examination of Hebbian-Covariance Rules and Spike TimingDependent Plasticity in Cat Visual Cortex in vivo. Front Synaptic Neurosci., 2: 147.

Frégnac, Y., \& Shulz, D. E. (1999) Activity-dependent regulation of receptive field properties of cat area 17 by supervised Hebbian learning. J Neurobiol., 41(1): 69-82.

Fritz, J., Shamma, S., Elhilali, M., \& Klein, D. (2003) Rapid task-related plasticity of spectrotemporal receptive fields in primary auditory cortex. Nat Neurosci., 6(11):1216-23.

Fu, Y., Kaneko, M., Tang, Y., Alvarez-Buylla, A., \& Stryker, M. P. (2015) A cortical disinhibitory circuit for enhancing adult plasticity. Elife, 4: e05558.

Gourévitch, B., Edeline, J.-M., Occelli, F., \& Eggermont, J. J. (2014). Is the din really harmless? Long-lasting effects of non-traumatic noise exposure on adult auditory cortex. Nature Reviews Neuroscience, 15: 483-491.

Hensch, T. K. (2005) Critical period plasticity in local cortical circuits. Nat Rev Neurosci., 6(11): 877-88.

Ihlefeld, A., Chen, Y. W., \& Sanes, D. H. (2016) Developmental Conductive Hearing Loss Reduces Modulation Masking Release. Trends Hear, 20: 233-65.

Kannan, M., Gross, G. G., Arnold, D. B., \& Higley, M. J. (2016) Visual Deprivation During the Critical Period Enhances Layer 2/3 GABAergic Inhibition in Mouse V1.J Neurosci., 36(22): 5914-9. 
Kotak, V. C., Fujisawa, S., Lee, F. A., Karthikeyan, O., Aoki, C., \& Sanes, D. H. (2005) Hearing loss raises excitability in the auditory cortex. J Neurosci., 25(15): 3908-18.

Kotak, V. C., Takesian, A. E., \& Sanes, D. H. (2008) Hearing loss prevents the maturation of GABAergic transmission in the auditory cortex. Cereb Cortex., 18(9):2098-108

Larimer, P., Spatazza, J., Espinosa, J. S., Tang, Y., Kaneko, M., Hasenstaub, A. R., Stryker, M. P., \& Alvarez-Buylla, A. (2016) Caudal Ganglionic Eminence Precursor Transplants Disperse and Integrate as Lineage-Specific Interneurons but Do Not Induce Cortical Plasticity. Cell Rep., 16(5): 1391-404.

McMahon, E., Wintermark, P., \& Lahav, A. (2012) Auditory brain development in premature infants: the importance of early experience. Ann N Y Acad Sci., 1252: $17-24$.

Milh, M., Kaminska, A., Huon, C., Lapillonne, A., Ben-Ari, Y., \& Khazipov, R. (2007) Rapid cortical oscillations and early motor activity in premature human neonate. Cereb Cortex., 17(7):1582-94.

Mowery, T. M., Kotak, V. C., \& Sanes, D. H. (2016) The onset of visual experience gates auditory cortex critical periods. Nat Commun., 7: 104-16.

Nichols, J. A., Jakkamsetti, V. P., Salgado, H., Dinh, L., Kilgard, M. P., \& Atzori, M (2007). Environmental enrichment selectively increases glutamatergic responses in layer II/III of the auditory cortex of the rat. Neuroscience, 145: 832-840.

Noreña, A. J., Gourévitch, B., Aizawa, N., \& Eggermont, J. J. (2006). Spectrally enhanced acoustic environment disrupts frequency representation in cat auditory cortex. Nat Neurosci, 9: 932-939.

Percaccio, C. R., Engineer, N. D., Pruette, A. L., Pandya, P. K., Moucha, R., Rathbun, D. L., \& Kilgard, M. P. (2005). Environmental enrichment increases paired-pulse depression in rat auditory cortex. J Neurophysiol, 94: 3590-3600.

Pi, H. J., Hangya, B., Kvitsiani, D., Sanders, J. I., Huang, Z. J., \& Kepecs, A. (2013) Cortical interneurons that specialize in disinhibitory control. Nature, 503(7477): $521-4$.

Pienkowski, M. \& Eggermont, J. J. (2009). Long-term, partially-reversible reorganization of frequency tuning in mature cat primary auditory cortex can be induced by passive exposure to moderate-level sounds. Hear Res, 257: 24-40.

Pienkowski, M. \& Eggermont, J. J. (2010). Passive exposure of adult cats to moderate-level tone pip ensembles differentially decreases AI and AII responsiveness in the exposure frequency range. Hear Res, 268: 151-162.

Pienkowski, M., Munguia, R., \& Eggermont, J. J. (2013). Effects of passive, moderate-level sound exposure on the mature auditory cortex: Spectral edges, spectrotemporal density, and real-world noise. Hear Res, 296: 121-130.

Pineda, R. G., Neil, J., Dierker, D., Smyser, C. D., Wallendorf, M., Kidokoro, H., Reynolds, L. C., Walker, S., Rogers, C., Mathur, A. M., Van Essen, D. C., \& Inder, T. (2014) Alterations in brain structure and neurodevelopmental outcome in preterm infants hospitalized in different neonatal intensive care unit environments. J Pediatr., 164(1): 52-60.

Polley, D. B., Heiser, M. A., Blake, D. T., Schreiner, C.E., \& Merzenich, M. M. (2004) Associative learning shapes the neural code for stimulus magnitude in primary auditory cortex. Proc Natl Acad Sci USA, 101(46): 16351-6. 
Rauschecker, J. P. (1991) Mechanisms of visual plasticity: Hebb synapses, NMDA receptors, and beyond. Physiol Rev., 71(2): 587-615.

Recanzone, G. H., Schreiner, C. E., \& Merzenich, M. M. (1993) Plasticity in the frequency representation of primary auditory cortex following discrimination training in adult owl monkeys. J Neurosci., 13(1): 87-103.

Rosen, M. J., Sarro, E. C., Kelly, J. B., \& Sanes, D. H. (2012) Diminished behavioral and neural sensitivity to sound modulation is associated with moderate developmental hearing loss. PLoS One, 7(7): e41514.

Sanes, D. H., \& Constantine-Paton, M. (1983) Altered activity patterns during development reduce neural tuning. Science, 221(4616): 1183-5.

Vanhatalo, S., Palva, J. M., Andersson, S., Rivera, C., Voipio, J., \& Kaila, K. (2005) Slow endogenous activity transients and developmental expression of $\mathrm{K}+-\mathrm{Cl}$ cotransporter 2 in the immature human cortex. Eur J Neurosci., 22(11): 2799-804.

Webb, A. R., Heller, H. T., Benson, C. B., \& Lahav, A. (2015) Mother's voice and heartbeat sounds elicit auditory plasticity in the human brain before full gestation. Proc Natl Acad Sci USA, 112(10): 3152-7

Weinberger, N. M. (2004) Specific long-term memory traces in primary auditory cortex. Nat Rev Neurosci., 5(4): 279-90.

Weinberger, N. M. (1995) Dynamic regulation of receptive fields and maps in the adult sensory cortex. Anпu Rev Neurosci., 18: 129-58.

Wickremasinghe, A. C., Rogers, E. E., Johnson, B. C., Shen, A., Barkovich, A. J., \& Marco, E. J. (2013) Children born prematurely have atypical sensory profiles.J Perinatol., 33(8): 631-5.

Wiesel, T. N. (1982) Postnatal development of the visual cortex and the influence of environment. Nature, 299(5884): 583-91.

Zhang, L. I., Bao, S., \& Merzenich, M. M. (2001). Persistent and specific influences of early acoustic environments on primary auditory cortex. Nat Neurosci, 4: 11231130.

Zheng, W. (2012). Auditory map reorganization and pitch discrimination in adult rats chronically exposed to low-level ambient noise. Front Syst Neurosci, 6: 65.

Zhou, X. \& Merzenich, M. M. (2012). Environmental noise exposure degrades normal listening processes. Nat Commun, 3: 843. 